\title{
Large-Area Ultrastructural Analysis on Alteration of Synaptic Vesicles in the 835MHz Radiofrequency-exposed Cerebral Cortex of Mice Brain Using Limitless Panorama and 3D Electron Tomography
}

Hyo-Jeong Kim ${ }^{1,2, \ddagger}$, Ju Hwan Kim ${ }^{2, \ddagger}$, Da-Hyeon $\mathrm{Yu}^{2}$, A Reum Je ${ }^{1}$, Suin Choi ${ }^{1,3}$, Hee-Seok Kweon ${ }^{1}$, Hak Rim Kim, ${ }^{2, *}$ and Yang Hoon Huh ${ }^{1, *}$

1. Center for Electron Microscopy Research, Korea Basic Science Institute, 162 Yeongudanji-ro, Cheongju-si, Chungbuk, Korea.

2. Department of Pharmacology, College of Medicine, Dankook University, 119 Dandaero, Cheonan-si, Chungnam, Korea.

3. Department of Biological Sciences and Biotechnology, College of Life Science and Nano Technology, Hannam University, Daejeon, Korea.

Despite the transmission electron microscope (TEM) provides tremendous ultrastructural information of cells and organelles [1], one of the disadvantages of TEM is the difficulty associated with observing large-area with high resolution [2 and 3]. Although several analytical techniques and equipments including ultramicrotomy-combined serial block face scanning EM have been developed recently to overcome such problem, those are mostly concentrated in SEM applications [1, 2, 4, 5, and 6]. In this research, to acquire large-volume TEM images with high-resolution from thick plastic section, we concomitantly used the 3D electron tomography and limitless panorama function in Shot Meister, a software suite that controls Bio-HVEM (JEM-1000BEF).

Exposure to radiofrequency electromagnetic fields (RF-EMFs) has become inevitable owing to widespread usage of mobile. Recently, numerous studies about the effects of mobile phone exposure have suggested influencing neuronal functions in the brain and thereby affecting behavior. Multiple enzymes are regulating the synthesis of neurotransmitters and synthesized neurotransmitters are stored in synaptic vesicles at axon terminal or synaptic boutons. Generally, the releasing of neurotransmitters from axon terminal is determined the function of neuron.

In the present study, we hypothesize that synaptic vesicles at axon terminals are affected by chronic exposure of RF-EMF exposure. Therefore, we apply 835MHz RF-EMF, SAR of 4.0W/kg for 5 hours daily for 4 weeks and 12 weeks to C57BL/6 mice and then we compared the synaptic vesicle size and numbers in 3D volume between control and RF-835MHz exposed mice. First, to check the synapse distribution, we used limitless panorama function and acquired large area panorama image by stitching the 81 tile images which acquired by combination of beam shift $(3 * 3)$ and gonio movement $(3 * 3)$ (Figure 1). Next, using the 3D electron tomography function, we focused on the 3D modeling of specific synapse region and on the statistical analysis of the synaptic vesicle number in pre-synaptic neurons (Figure 2). Of interest, the number and size of synaptic vesicles are slightly decreased in synaptic boutons of neuron at cerebral cortex after exposure of RF-EMF. This result indicates that the amounts of neurotransmitters of neuron are changed at cerebral cortex after exposure of $835 \mathrm{MHz}$ RF-EMF through altered profiles of synaptic vesicles in neuron. Also, this result clearly demonstrates the contribution of 3D electron tomography and limitless panorama-based large-area Bio-HVEM image analysis system for the acquisition of high throughput statistical information for an accurate interpretation of various morpho-functional alterations of cellular organelles [7]. 
References:

[1] K Miranda et al., Mol. Reprod. Dev. (2015), doi: 10.1002/mrd. 22455.

[2] E Oho et al., Journal of Electron Microscopy 49 (2000), p.135-141.

[3] Y Ikeda et al., Microsc. Microanal. 19 (2013), p.1330-1331.

[4] C Blumer et al., Medical Image Analysis 19 (2015), p.87-97.

[5] H Horstmann et al., Plos ONE 7 (2012), e35172.

[6] LCS Medeiros et al., Plos ONE 7 (2012), e33445.

[7] This research was supported by Korea Basic Science Institute grants (\#T37416).

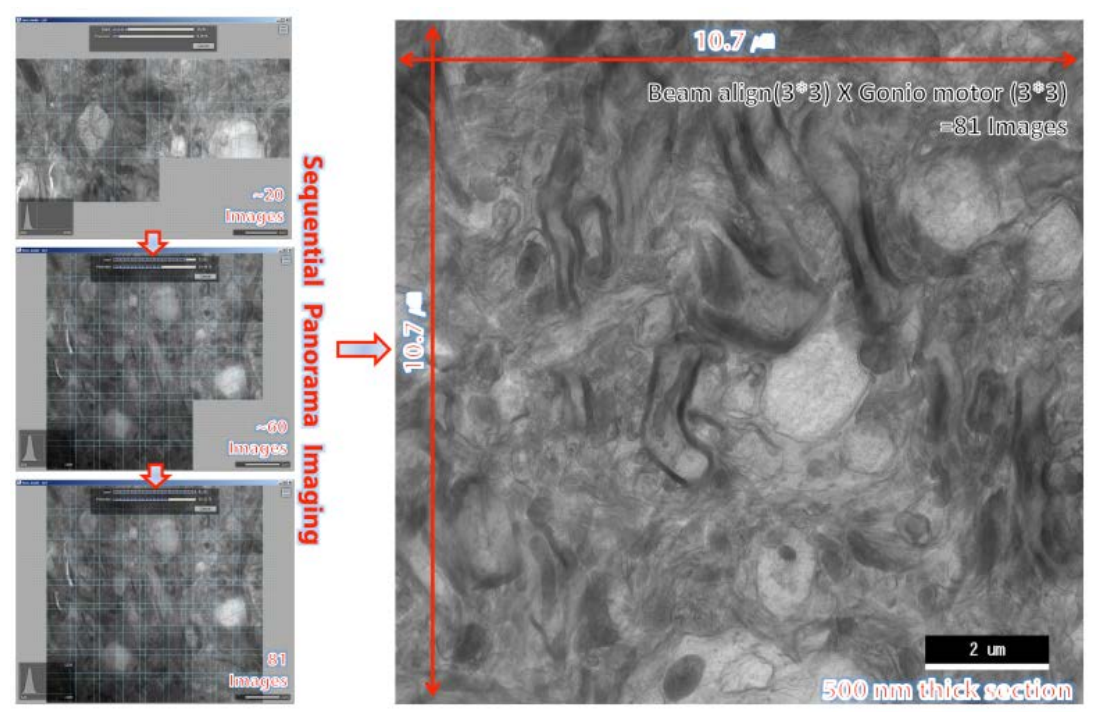

Figure 1. Sequential panorama imaging procedure for the Enhanced large-area HVEM image acquisition from thick section by using Shot Meister program of Bio-HVEM(JEM-1000BEF).

\section{a. control}

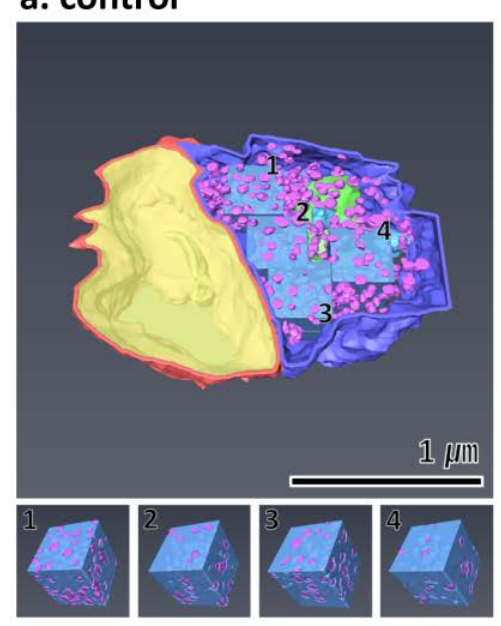

$0.2 \mu \mathrm{m}$

\section{b. $\mathrm{RF}-835 \mathrm{MHz}$}

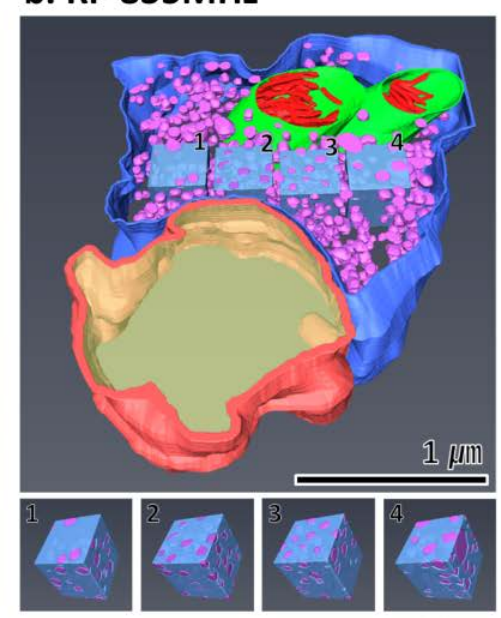

\section{c. Statistical graph}

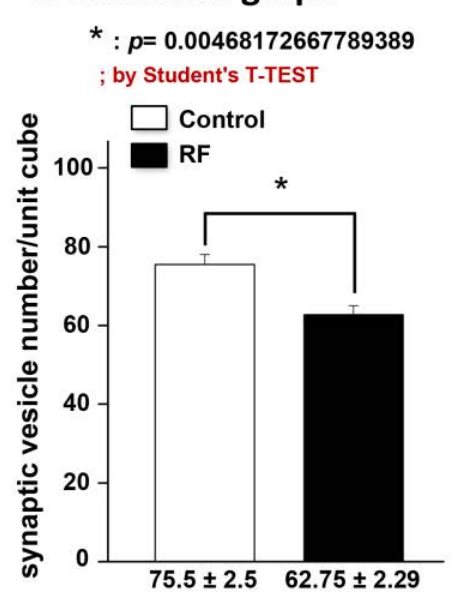

$0.2 \mu \mathrm{m}$

Figure 2. 3D models of synapse region of control (a) and RF-835 exposed (b) mice by 3D electron tomography and statistical analysis of synaptic vesicles in 3D volume of pre-synaptic neuron (c). 